\title{
Shared-Autonomy Control for Intuitive Bimanual Tele-manipulation
}

\author{
Marco Laghi ${ }^{1 *}$, Michele Maimeri ${ }^{1 *}$, Mathieu Marchand ${ }^{4}$, Clara Leparoux $^{4}$, \\ Manuel G. Catalano ${ }^{1}$, Arash Ajoudani ${ }^{2}$ and Antonio Bicchi ${ }^{1,3}$
}

\begin{abstract}
In this work we propose a framework for bimanual teleoperation that includes two control strategies: (i) the classic one-to-one coupling of the human and robotic arms (which allows to fully exploit the user dexterity) and (ii) a new shared autonomy strategy, in which the two robotic arms are controlled through the movements and gestures of just one user's arm (relaxing the user's cognitive load). Moreover, the use of tele-impedance allows the user to also control the remote physical interaction.

Index Terms-Teleoperation, bimanual manipulation, shared autonomy
\end{abstract}

\section{INTRODUCTION}

The existing dual-arm teleoperation systems function on one-to-one coupling of the human and robotic arms with the aim to increase the dexterity of bimanual tele-manipulation. This provides the users with the ability to individually control each robot end-effector's motion, to successfully execute asymmetric bimanual tasks (see e.g. [1]-[3]). While the independent control of the robot end-effectors are necessary in executing complex and asymmetric tasks, it can result inconvenient in symmetric bimanual tasks, especially in absence of force feedback. For instance, while holding and carrying a heavy load using two arms, an individual control of the robot movements may impose a high cognitive load, particularly to compensate for non perfect human-robot movement coupling.

Hence, we developed a novel framework that integrates a shared autonomy tele-manipulation interface to enable human operators to effectively and comfortably achieve symmetric bi-manual tasks. This is done by the recognition of human hand-arm gestures (hand fist and finger spread) in real-time, to implement an independent or a synergistic mode (Fig. 1) to control the movement and the physical interaction performance of the bimanual robotic system. The latter is performed through the tele-impedance paradigm [4], i.e., the remote control of robot impedance and position references. Preliminary results confirm the improved intuitiveness, acceptability and performance of the shared-autonomy teleoperation framework.

This work was supported by the European Commission projects (Horizon 2020 research program) SOMA (no. 645599)

*These authors contributed equally to this work.

${ }^{1}$ Soft Robotics for Human Cooperation and Rehabilitation (SoftBots), Istituto Italiano di Tecnologia, Genoa, Italy

${ }^{2}$ Human-Robot Interfaces and Physical Interaction (HRI2), Istituto Italiano di Tecnologia, Genoa, Italy

${ }^{3}$ Centro di Ricerca "E. Piaggio", Universita di Pisa, Largo L. Lazzarino, 1 , 56126 Pisa, Italy.

${ }^{4}$ Département Électronique, Électrotechnique, Automatique (EEA), Ecole Normale Supérieure Paris Saclay, 61, av Président Wilson F-94520-Cachan, France.

Correspond to: \{marco.laghi, michele.maimeri\} at it.it

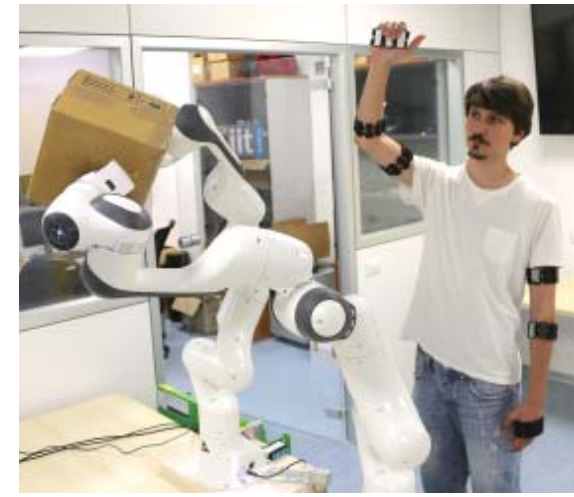

Fig. 1. An example frame of single-arm synergistic manipulation operated with the proposed approach.

\section{METHODS AND MATERIALS}

The proposed framework can be divided into two well distinguished parts: the first one concern the human inputs, the second regards how these inputs are used for the implementation of the two control strategies and the switch between them. An explanation of the two parts follows hereafter.

\section{A. Human inputs}

The desired control strategies require three inputs from the user, all in real time: 1) the user's hands position and 2) the user's arm impedance trend, both used as references for the tele-impedance control of the robotic counterparts; and 3) the hand gesture for the robot control and the switching between the two strategies (see Subsec. II-B). All these three inputs are retrieved using Myo armbands [5], which encapsulate inertial (IMU) and electromyography (EMG) sensors. Six Myo armbands in total are used (see Fig. 1): two for the upper arms, two for the forearms and two for the hands. Knowing a

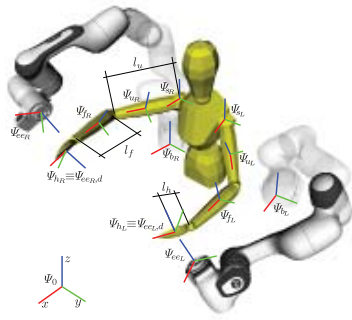

(a)

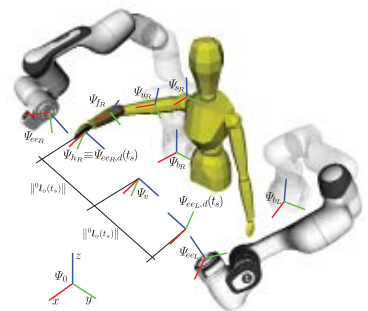

(b)
Fig. 2. Representation of the frames involved in (a) the independent strategy and (b) the synergistic strategy. The user is virtually positioned in the middle of the dual-arm robotic platform. In (b) the frames of the user's left hand are not depicted because not involved in this strategy. 

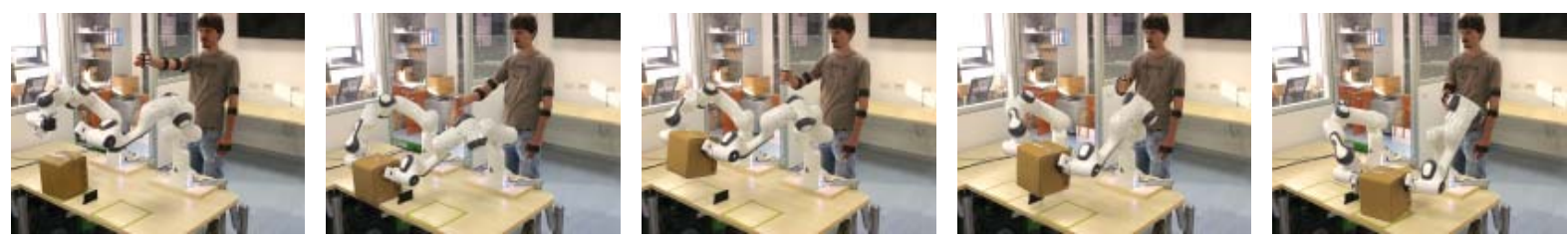

Fig. 3. Photo sequences of the pick and place task.

priori the arm segment lengths $\left(l_{u}, l_{f}\right.$ and $l_{h}$, see Fig. 2(a)), the position and orientation of each hand are calculated using the IMUs embedded in the Myos. The user's arm stiffness indexes are retrieved monitoring the upper arm's EMG Myos, similar to [6]. The hands gestures are instead recognized monitoring the EMGs of the forearm muscles, through which we can recognize a fist or a finger spread gesture.

\section{B. Control strategies}

1) Independent dual-arm control strategy: This is the classic strategy used for bimanual tele-manipulation. Each robotic arm is commanded independently, depending on the movements of the operator arms (Fig. 2(a)). The robots are controlled through tele-impedance paradigm [4], an impedance control where the reference position and the impedance gains are the estimated user's arm ones (Subsec. II-A).

2) Synergistic single-arm control strategy: In this strategy, the two robotic arms move following only one user's arm, namely the right. The strategy is activated when the operator perform a left finger spread in the independent strategy. From that moment, a virtual frame $\Psi_{v}$ rigidly linked to the right hand and oriented as this last is created in the middle point between the two robot (Fig. 2(b)). Then, the distance $l_{o}$ between the right hand frame $\Psi_{h_{R}}$ and $\Psi_{v}$ is initially calculated, and used to create the robots reference frames that always face each other, with the right one oriented as the right hand. $l_{o}$ is then varied by the user through right hand gestures: a fist gesture decrease it, a finger spread increase it. As soon as another left finger spread gesture is performed, the control switch back to independent one.

\section{EXPERIMENTS}

To assess the validity of the new strategy, we used two Panda arms by Franka Emika [7], as shown in Figs. 1 and 2. Once the user wear the armbands, a brief calibration on the IMUs and EMGs is performed. After that, the system is ready. We asked to five naive subjects to perform three different manipulation tasks of a box, all of them with both the independent and the synergistic strategies. The tasks were: 1) pick and place (Fig. 3), 2) box rotation and 3) box tilting.

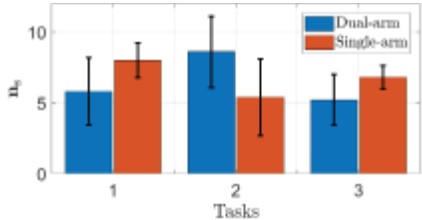

Fig. 4. Average succeded trials $n_{s}$ of the three experimental tasks and their standard deviation for both control strategies: blue for the dual-arm and orange for the single-arm.
Before each task-control strategy combination, participants performed one minute of training to become familiar with the system. We evaluated the number of actions/movements $n_{s}$ correctly performed (box not fallen) in a period of 2 minutes for each task. Results are shown in Fig. 4.

\section{RESUlTS AND Discussion}

Fig. 4 results suggest that the subjects performed better with the proposed synergistic strategy than the dual-arm in the first and the third tasks. Furthermore, in these two cases the variance registered for the dual-arm strategy is consistently larger than for the single-arm. This last observation seems to suggest that the performances achieved with the dual-arm strategy are strongly user-dependent, while the single-arm strategy is able to compensate in part for the user's lack of experience and dexterity. Direct discussions with the subjects confirms that their confidence in using one strategy or another depends on the task to be performed.

\section{Conclusions}

A two levels autonomy framework for bimanual telemanipulation is here proposed. With this architecture, the user can choose between the classic independent control and a new synergistic control strategy, in which the robots move in a coordinated way. The provided preliminary results suggested that both strategies are useful, depending on the desired task.

Future works will focus on an extensive evaluation of the proposed strategy, involving an higher number of subjects and tasks, and the possibility of increasing the number of autonomy levels among which the user can choose.

\section{REFERENCES}

[1] A. Peer, Y. Komoguchi, and M. Buss, "Towards a mobile haptic interface for bimanual manipulations," in Intelligent Robots and Systems, 2007. IROS 2007. IEEE/RSJ International Conference on. IEEE, 2007, pp. 384-391.

[2] F. Ferraguti, N. Preda, M. Bonfe, and C. Secchi, "Bilateral teleoperation of a dual arms surgical robot with passive virtual fixtures generation," in Intelligent Robots and Systems (IROS), 2015 IEEE/RSJ International Conference on. IEEE, 2015, pp. 4223-4228.

[3] T. Hulin, K. Hertkorn, P. Kremer, S. Schätzle, J. Artigas, M. Sagardia, F. Zacharias, and C. Preusche, "The dlr bimanual haptic device with optimized workspace," in Robotics and Automation (ICRA), 2011 IEEE International Conference on. IEEE, 2011, pp. 3441-3442.

[4] A. Ajoudani, C. Fang, N. Tsagarakis, and A. Bicchi, "Reducedcomplexity representation of the human arm active endpoint stiffness for supervisory control of remote manipulation," The International Journal of Robotics Research, vol. 37, no. 1, pp. 155-167, 2018.

[5] "Myo armband," https://www.myo.com/.

[6] M. Laghi, A. Ajoudani, M. Catalano, and A. Bicchi, "Tele-impedance with force feedback under communication time delay," in Intelligent Robots and Systems (IROS), 2017 IEEE/RSJ International Conference on. IEEE, 2017, pp. 2564-2571.

[7] "Franka emika," https://www.franka.de/. 